\title{
Optimization of AEB Key Parameters in Car to Electric-Two-Wheeler Accidents
}

\author{
Sumin Tang \\ Shanghai University of Engineering Science, Shanghai, China \\ Email: 1518206411@qq.com
}

How to cite this paper: Tang, S.M. (2018) Optimization of AEB Key Parameters in Car to Electric-Two-Wheeler Accidents. Open Access Library Journal, 5: e4754. https://doi.org/10.4236/oalib.1104754

Received: July 4, 2018

Accepted: July 21, 2018

Published: July 24, 2018

Copyright $\odot 2018$ by author and Open Access Library Inc.

This work is licensed under the Creative Commons Attribution International License (CC BY 4.0).

http://creativecommons.org/licenses/by/4.0/

\begin{abstract}
In order to determine the optimal value of key parameters of automatic emergency braking (AEB) system, an optimization method based on real accident simulation is proposed. Accident reconstruction of 65 car to electric-two-wheeler collision accidents was carried out by using PC-Crash. According to the different parameter values of AEB system radar, the virtual accident reconstruction results are obtained. The optimal value of key AEB parameters was determined by comparing the accident avoidance rate. The results show that: based on these 65 real rear end collisions, the final optimization results of the radar detection angle and detection distance in the AEB system are $90^{\circ}$ and $120 \mathrm{~m}$ respectively.
\end{abstract}

\section{Subject Areas}

Mechanical Engineering

\section{Keywords}

AEB, Car to Electric-Two-Wheeler Accident, Radar, Key Parameters, Simulation, Optimization

\section{Introduction}

In recent years, the sales volume and ownership of electric two-wheeled vehicles continue to increase [1], which not only aggravates the current situation of traffic congestion, but also leads to frequent accidents of electric two-wheeled vehicles and the consequences are often very serious. Research shows that under dangerous conditions, if the driver has $0.5 \mathrm{~s}$ of pretreatment time, the collision accident can be reduced by 30\% [2]. Automatic Emergency Braking (AEB) system can issue an alarm and implement braking intervention before the occur- 
rence of a collision to minimize the occurrence or damage [3]. Foreign scholars have studied the performance and utility of AEB system by using specific cases and depth data of traffic accidents, aiming at developing AEB system which is suitable for its own road environment and traffic conditions through simulation test. American scholar [4] [5] establishes a typical accident case through an independent accident case study and proposes a AEB system for the analysis of rear end collision accidents based on the database of National Automotive Sampling System (NASS) and vehicle collision data system (Crashworthiness Data System, CDS) in the United States. Australian scholars [6] conducted in-depth studies on accidents and evaluated the potential utility of AEB system based on accident reconstruction and simulation. German scholars [7] [8] [9] based on the German In-Depth Accident Study (GIDAS) database, evaluated the potential utility of automobile active safety technology through accident simulation. Domestic researches on AEB system mainly focus on simulation test and control strategy. Lijun Jiang et al. [10] combined the real dangerous working conditions and accident working conditions data collected by the driving recorder in China to analyze and obtain the test conditions of AEB for rear-end avoidance. Xiaofei Dong et al. [11], based on the real dangerous working data in China, obtained the characteristics of the driver's reaction time, braking speed reduction, collision time (Time To Collision, TTC), workshop time (Time Headway, THW), TTC $^{-1}$ (TTC reciprocal) and other parameters under dangerous working conditions. However, there is no AEB system applicable to China's road traffic environment and conditions in China. In this paper, the algorithm and model of AEB system are established based on the specific case of the collision between cars and electric two-wheeled vehicles in China's road traffic environment. Combined with the accident reconstruction data of PC-Crash, the performance and utility of AEB system were investigated in each accident case, and the key parameters of AEB system were studied with radar parameters as an example, to determine the optimal parameter combination of AEB system for China's road traffic environment and provide references for the development of AEB system for the Chinese market.

\section{Data Basis}

The accident cases involved in this paper come from the data collected by the National Automobile Accident In-depth Investigation System (NAIS) In the Songjiang area in the past five years (see Table 1). The basic form of these cases, the damage characteristics and collision speed is analyzed, and car-electric two rounds of the typical working condition of the car collision accident, for subsequent AEB system key parameters and utility of the study provides basic data support.

In order to screen out typical car-electric two wheel vehicle accident conditions, this paper mainly statistics the accident location, collision form and collision area, in order to explore its distribution characteristics. Through the 
Table 1. Car to electric-two-wheeled vehicle typical accident condition.

Accident condition
$\begin{aligned} & \text { Example of accident } \\ & \text { The car goes straight, } \\ & \text { the electric two wheeled } \\ & \text { vehicle enters from the } \\ & \text { right side (A1). }\end{aligned}$ $\begin{aligned} & \begin{array}{l}\text { Case } \\ \text { ratio }\end{array} \\ & \text { The cause of the depth of the accident } \\ & \begin{array}{l}\text { The car goes straight, } \\ \text { the electric two wheeled } \\ \text { vehicle moves from the } \\ \text { left to the left (B1). }\end{array}\end{aligned}$

classification and statistics of the basic information of the accident cases, the distribution map of the accident location (Figure 1) and the distribution map of the collision pattern are obtained (Figure 2).

As you can see from Figure 1, car to electric-two-wheel accidents occur at intersections: $53.85 \%$ at crossroads and $21.54 \%$ in T-junction accidents. Observation Figure 2 shows that the collision of cars and electric two wheels is mainly the side collision of the car and the electric two wheels, that is, the side of the electric two wheels on the front of the car. According to Figure 1, Figure 2, we can see that the accident pattern is mainly the side collision accident at the intersection.

In order to study the collision area specifically, as shown in Figure 3, in the 65 car-electric bicycle cases, the ratio of collision to the left or right side of the car is about $23 \%$ and $25 \%$, and the proportion of the collision with the front of the car is about $52 \%$ and the largest proportion. In Figure 4, there were 62 accidents in the green markings, about $95 \%$. According to the above analysis, the following two typical accidents of car electric two wheeled vehicle are shown in Table 1.

\section{Simulation Research Scheme}

PC-Crash is an application software for accident reconstruction and reconstruction using momentum and impulse. This paper chooses the software to carry out simulation research. First of all, the PC-Crash software is used to reconstruct the accident cases in 65 cases (see Figure 5) to obtain the motion response and movement process of the vehicle before the collision, the collision moment and after the collision, and reproduces the accident process. Secondly, each case is simulated again through the modification of AEB system parameters, and the virtual accident result is obtained (see Figure 6). By comparing the parameters of the radar to avoid the utility of the accident, the optimum parameters of the radar parameters of the AEB system are determined. In the study of the key parameters of the AEB system, the accident avoidance rate under the system is used as the evaluation condition of the AEB system, and the optimal value of the 


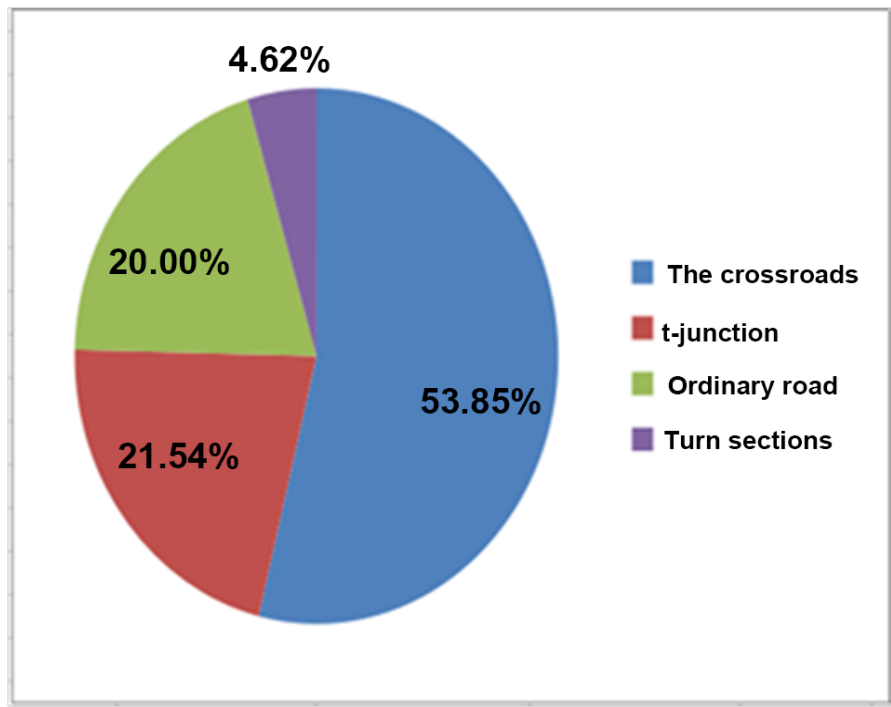

Figure 1. Distribution of accident location.

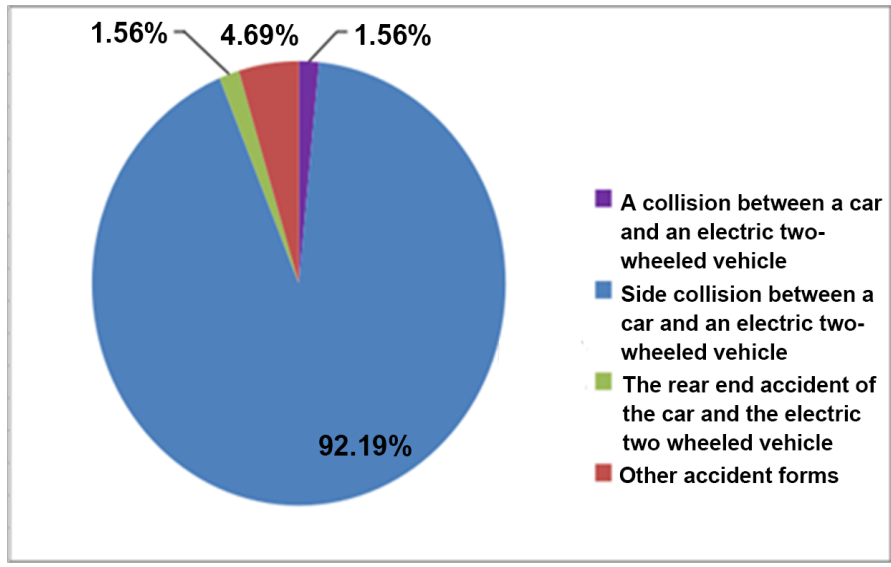

Figure 2. Collision shape distribution.

$23 \% \quad(n=15)$

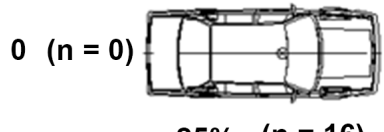

$52 \%(n=34)$

$25 \% \quad(n=16)$

Figure 3. The overall collision area of the car.

$100 \%(n=65)$

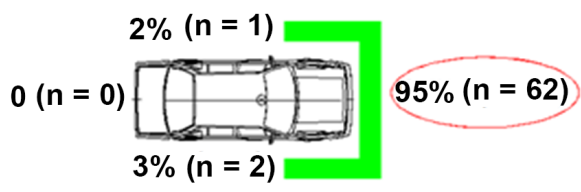

Figure4. Car specific collision area. 


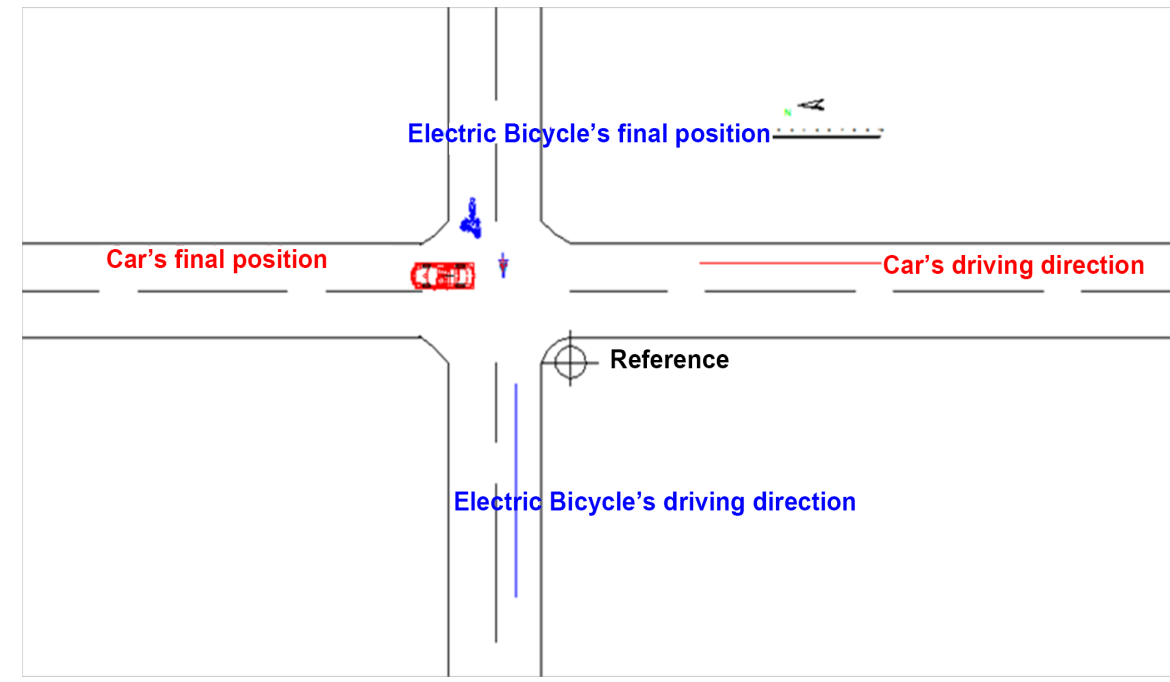

Figure 5. PC-Crash accident reconstruction results.

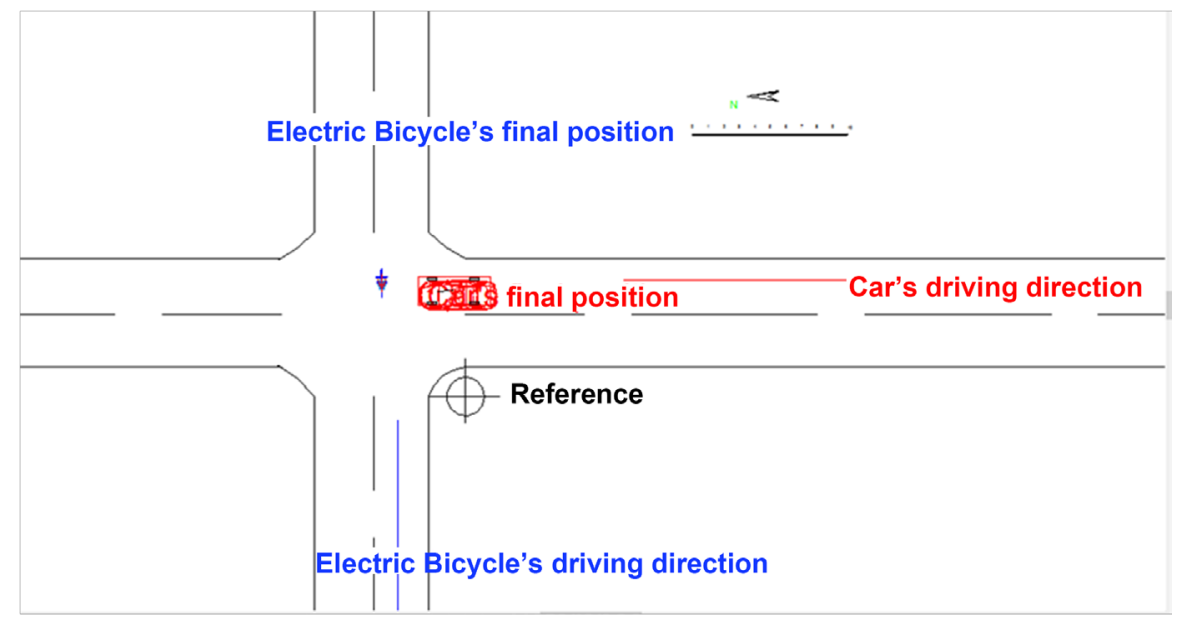

Figure 6. PC-Crash simulation results after modifying the parameters of AEB system.

key parameters is obtained by the collision avoidance performance of the AEB system as a measure. The rate of accident avoidance is defined as:

Accident avoidance rate

$$
=\frac{\text { The number of the AEB system to avoid accidents successfully }}{\text { Total number of accidents }} \times 100 \%
$$

The use of multistage braking can help the AEB system to intervene more comfortably and smoothly in dangerous conditions. The braking effect is stronger, the rate of speed decreases is greater, and the performance of collision avoidance is better than single stage braking [12]. Therefore, the AEB system adopts the two stage braking strategy, referring to the maximum adhesion coefficient of the general pavement, the partial braking level reduction of typical car to human AEB system in the PreScan manual, and the typical AEB system parameter setting in the market, and considering the effect of minimizing the impact of TTC on the simulation of radar parameters, setting the AEB two. The 
brake part parameters are shown in Table 2 (TTC corresponding to the two level brake is TTC1 and TTC2 respectively). In actual simulation, the deceleration of vehicles is restricted by the actual road adhesion conditions in each sample.

In the process of radar detection, the greater the detection angle and detection distance, the greater the impact on the detection quality and detection efficiency, and the higher the possibility of generating error signals and interference. At the same time, the setting of detection angle and detection distance also needs to be realized within the actual radar detection technology. In this paper, based on the existing radar products on the market, within the range of detection technology can support maximum play to the AEB collision avoidance performance of the system as the principle, the radar detection Angle and distance were analyzed, and the two key parameters to determine the optimal values of key parameters of radar.

The key parameters of the typical radar are shown in Table 3, so that the key parameters range of the existing products can be understood. The values of the set angle are 10, 20,30, 40, 50 and 90, respectively. The detection angle of 90 degrees can only be achieved within the detection distance of less than $60 \mathrm{~m}$ in the actual radar products. It does not have enough detection ability for a long distance. The results of the simulation can only be used as comparison and reference. The setting range of detection is $40 \mathrm{~m}, 60 \mathrm{~m}, 80 \mathrm{~m}, 100 \mathrm{~m}, 120 \mathrm{~m}, 150 \mathrm{~m}$, $180 \mathrm{~m}, 210 \mathrm{~m}$ and $250 \mathrm{~m}$ respectively.

For the detection angle and detection distance, control variables and separate research methods are adopted to discuss their impact on collision avoidance respectively. The simulation process, as shown in Figure 5, shows the optimal values of detection angles and detection distances obtained in the simulation process as RTA (Radar Test Angle) and RTD (Radar Test Distance), RTD initial value RTD $(0)=100 \mathrm{~m}$ (the intermediate value of the range of radar product detection range in the market).

\section{Analysis of Simulation Results}

\section{1) Results}

According to the flow chart shown in Figure 7, the optimal combination of radar parameters is $\mathrm{RTD}=120 \mathrm{~m}, \mathrm{RTA}=90^{\circ}$, and the simulation ends.

\section{2) DISCUSSION}

$\mathrm{RTD}=120 \mathrm{~m}$ was set to simulate the radar detection angle.

Table 2. Parameter setting of two-stage brake.

\begin{tabular}{cc}
\hline Project & set value \\
\hline Emergency brake level reduction $/ \mathrm{m} \cdot \mathrm{s}^{-2}$ & 7.5 \\
Partial brake level reduction $/ \mathrm{m} \cdot \mathrm{s}^{-2}$ & 3.5 \\
TTC1/s & 2.0 \\
TTC2 $/ \mathrm{s}$ & 1.5 \\
\hline
\end{tabular}


Table 3. Summary of key parameters of typical radar.

\begin{tabular}{cccc}
\hline brand & Radar products & $\begin{array}{c}\text { Detection } \\
\text { angle } /\left(^{\circ}\right)\end{array}$ & $\begin{array}{c}\text { Detection } \\
\text { distance/(m) }\end{array}$ \\
\hline \multirow{2}{*}{ Bosch } & Medium range radar sensor (MRR) & 42 & 160 \\
& Long-range radar sensor (LRR) & 30 & 250 \\
Delphi & $\begin{array}{c}\text { Electronic scanning radar (ESR) Medium } \\
\text { distance large angle mode }\end{array}$ & 90 & 60 \\
& ESR Remote small angle mode & 20 & 174 \\
\hline
\end{tabular}

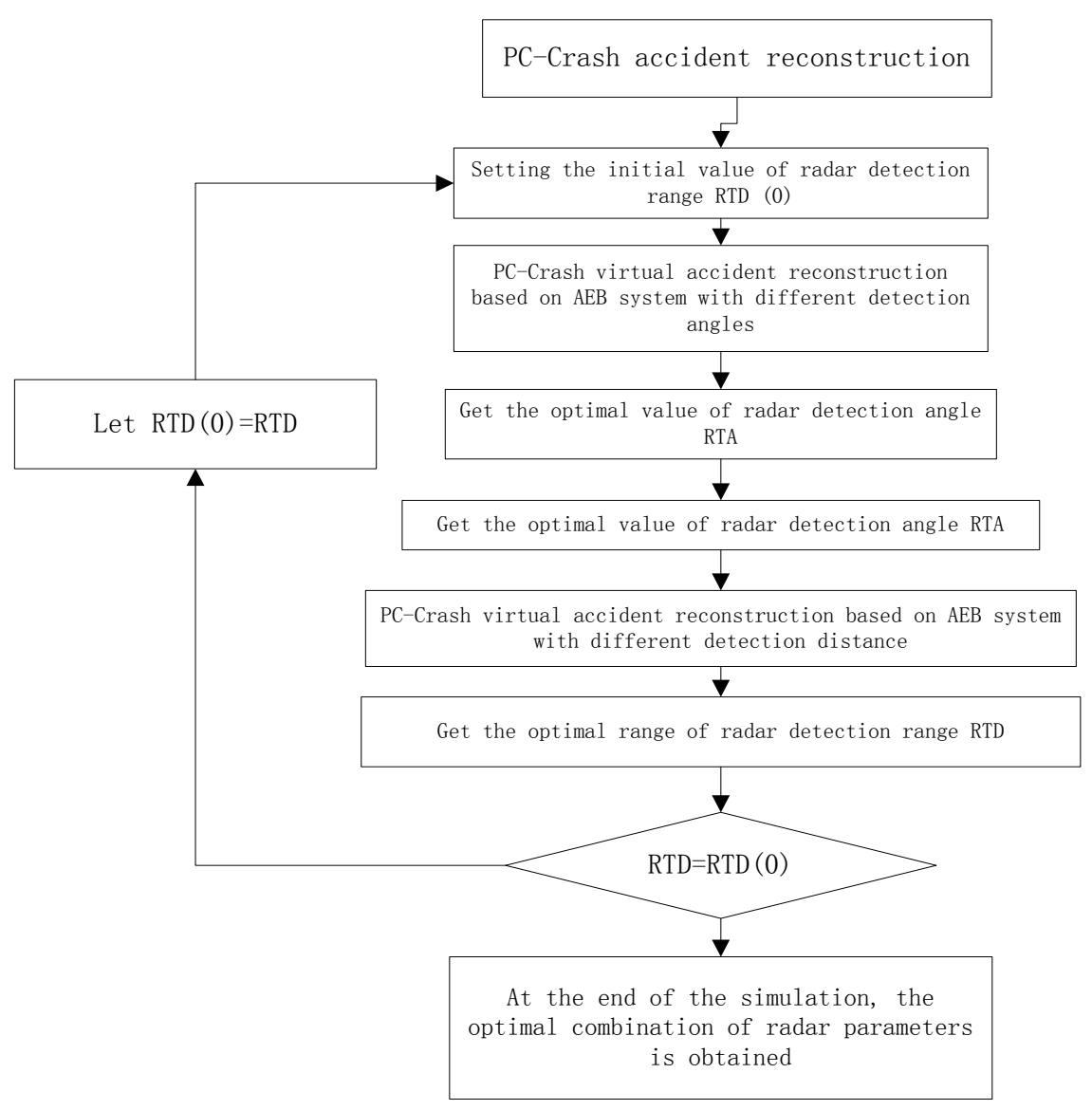

Figure 7. Simulation flow chart.

The accident avoidance rate of each typical working condition under different detection angles is shown in Figure 8. Along with the rising of the detection Angle, for overall accident collision avoid rate increased from $81.8 \%$ to $93.6 \%$ (RTA $=90^{\circ}$ ) Figure 6 different detection angle under the conditions of various typical working conditions to avoid accident rate.

\section{1) Results}

When the detection distance is set to an ideal value of $120 \mathrm{~m}$ and the detection angle increases from 10 to 90 degrees, the overall avoidance rate gain of the accident is 11.8 percentage points (from $81.8 \%$ to $93.6 \%$ ), while the detection angle increases from 10 to 90 , so the optimal value of the probe angle is 90 . 


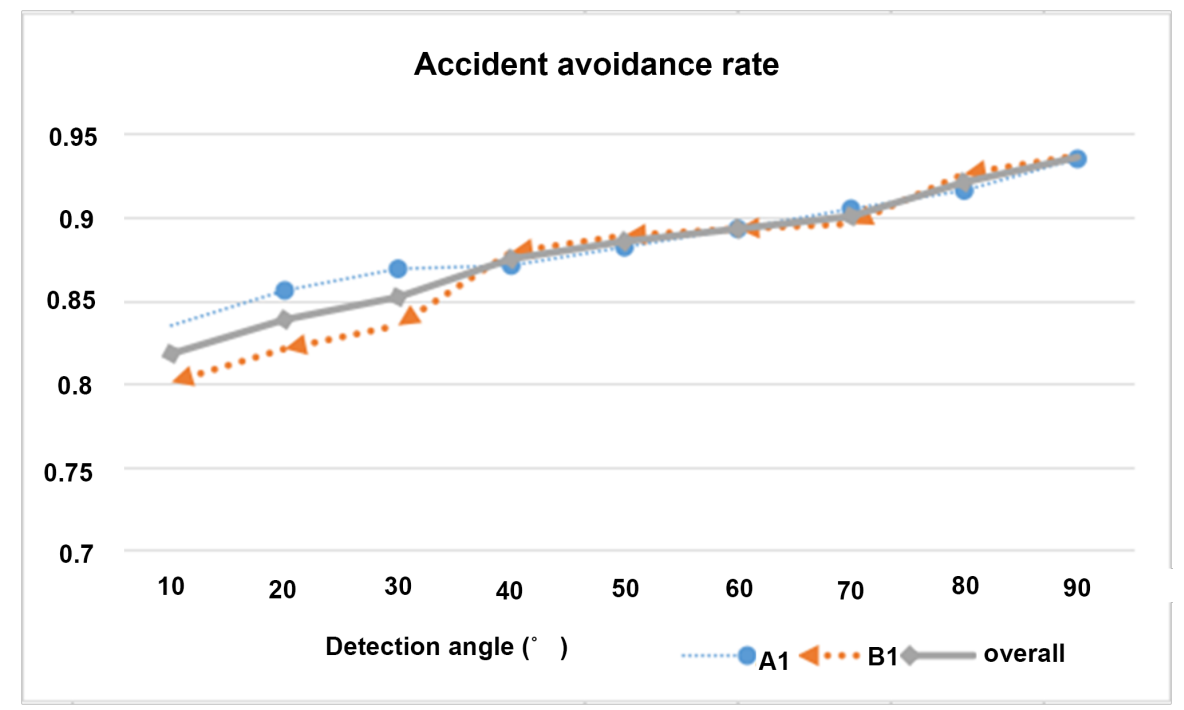

Figure 8. Accident avoidance rate of typical working conditions under different detection angles.

\section{2) Discussion}

Set RTA $=40$ degrees to simulate the detection distance.

The accident avoidance rate of each typical working condition at different detection distances is shown in Figure 9. When the detection Angle is $90^{\circ}, 120 \mathrm{~m}$ maximizes the AEB under the detection range of collision avoidance performance of the system. When the detection distance reaches $120 \mathrm{~m}$, the increase of detection distance will no longer generate gain for the overall collision avoidance rate and accident avoidance rate of various typical accident conditions. Figure 7 accident avoidance rates at different detection distances under typical operating conditions.

When the detection Angle to achieve the optimal value of $90^{\circ}, 120 \mathrm{~m}$ of the detection range will still be able to play to the greatest extent under different conditions the collision avoidance of the AEB system performance, and get the optimal value detection Angle $90^{\circ}$ set the detection range of initial value. Therefore, the optimal radar detection distance in AEB system is set at $120 \mathrm{~m}$.

So far, the optimal value of the key parameters of the radar has all been produced. Under such parameter setting, the collision avoidance performance of AEB system can be maximized in different typical working conditions, which is the optimal parameter combination obtained from the study of radar key parameters.

\section{Conclusions}

This paper analyzes the characteristics of 65 cars to electric two wheelers collision accidents, and uses PC-Crash to reconstruct accidents. On this basis, taking the radar parameters as an example, the AEB system is intervened under different parameters, and the virtual reconstruction results of each accident are re-obtained. Through the analysis of the accident avoidance rate after AEB 


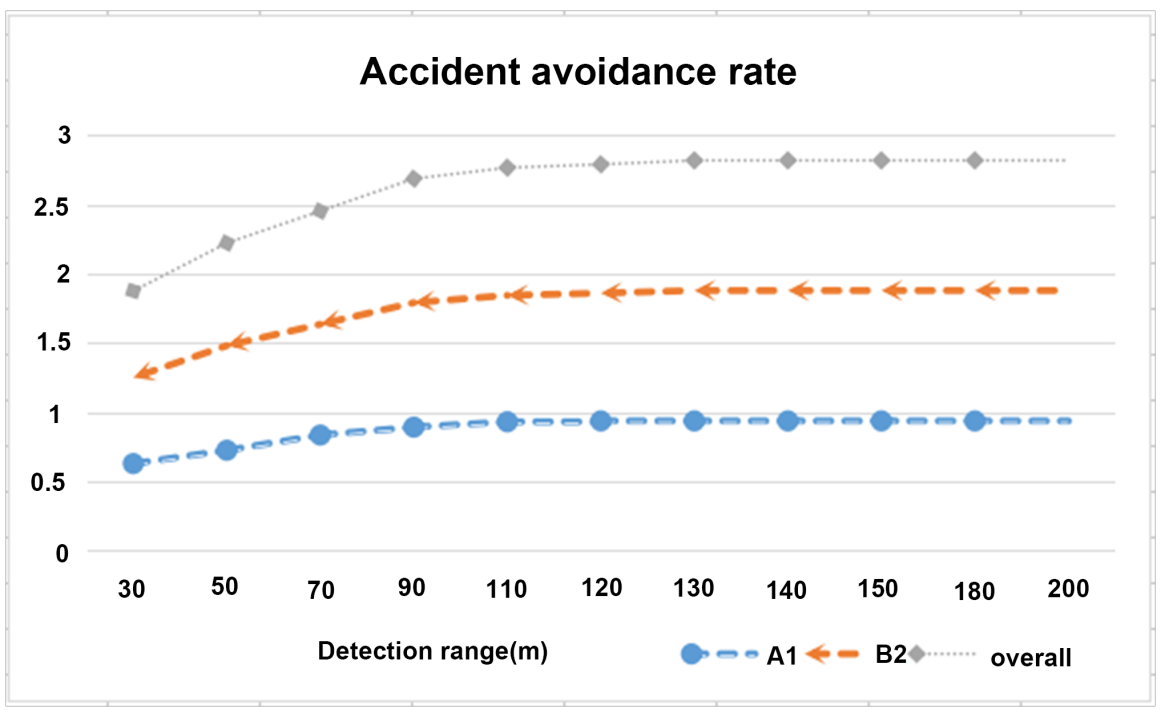

Figure 9. Accident avoidance rate of typical accident conditions under different detection range.

system intervention under different parameters, the optimized combination of radar detection angle and detection distance of AEB system is 90 and $120 \mathrm{~m}$, and the overall accident avoidance rate of the accident is $93.6 \%$ under the combination of the parameters.

In the research process, the sample size is small, the influence of weather factors on the sensor model is not considered, and the driver model is not added. In the further study, the acquisition range and the rich sample capacity will be expanded, and the driver model and the AEB system early warning function will be added to the driver police. The result is more accurate.

\section{References}

[1] Xu, X., Chen, J.Y. and Da, C. (2014) Analysis of Side Impact Accidents Based on Deep Accident Research. 2014 Seventeenth China International Symposium on Automotive Safety Technology, Baoding, 24-32.

[2] Liu, M.Z. (2006) Research on Rear End Collision Warning System for Freeway. Wuhan University of Technology, Wuhan.

[3] Active Test Consortium (2011) Dissemination of Performance Testing Methods for Active Safety Functions in Road Vehicles.

[4] Kusano, K.D. and Gabler, H.C. (2010) Potential Occupant Injury Reduction in PreCrash System Equipped Vehicles in the Striking Vehicle of Rear-End Crashes. 54th AAAM Annual Conference Annals of Advances in Automotive Medicine, Las Vegas, 189-193.

[5] Kusano, K.D. and Gabler, H.C. (2012) Safety Benefits of Forward Collision Warning, Brake Assist and Autonomous Braking Systems in Rear-End Collisions. IEEE Transactions on Intelligent Transportation Systems, 13, 1546-1555. https://doi.org/10.1109/TITS.2012.2191542

[6] Anderson. R., Doecke, S., Mackenzie, J., et al. (2013) Potential Benefits of Autonomous Emergency Braking Based on In-Depth Crash Reconstruction and Simulation. International Technical Conference on the Enhanced Safety of Vehicles (ESV), 
Seoul, 36-75.

[7] Erbsmehl, C. (2009) Simulation of Real Crashes as a Method for Estimating the Potential Benefits of Advanced Safety Technologies. International Technical Conference on the Enhanced Safety of Vehicles (ESV), Stuttgart, 65-73.

[8] Lindman, M. and Tivesten, E. (2006) Method for Estimating the Benefit of Autonomous Braking Systems Using Traffic Accident Data. SAE World Congress, Detroit.

[9] Georgi, A., Zimmermann, M., Lich, T., et al. (2009) New Approach of Accident Benefit Analysis for Rear End Collision Avoidance and Mitigation Systems. International Technical Conference on the Enhanced Safety of Vehicles, Stuttgart, 78-95.

[10] Jiang, L.J. (2014) Research on the Evaluation Method of Automatic Emergency Braking System. Tongji University, Shanghai, 11.

[11] Dong, X.F. (2013) Research on Accident Avoidance Strategy in Typical Dangerous Conditions. Tongji University, Shanghai.

[12] Dai, C. (2015) Key Parameters and Utility Evaluation of Pedestrian Collision Avoidance Safety System. Tongji University, Shanghai. 